\title{
REHABILITACIÓN ORAL CON PROSTODONCIA PARCIAL FIJA: REPORTE DE UN CASO COMPLEJO DE ARCO CRUZADO
}

\author{
Juan Carlos Cabrera Rojas \\ Odontólogo U. Santo Tomás, Estudiante de III año Especialización en Rehabilitación Oral \\ U. Santo Tomás, Bucaramanga (Colombia)
}

Correo electrónico: juanc_1970@yahoo.com.mx

\begin{abstract}
RESUMEN
Es un reto para la rehabilitación devolver la función y la estética perdida al sistema estomatognático. En el caso de tratamientos complejos, la rehabilitación oral juega un papel clave en el diagnóstico y vela para que se cumplan los objetivos propuestos dentro de parámetros académicos y prácticos. En este reporte se presenta un caso clínico de características complejas rehabilitado mediante prostodoncia parcial fija que requirió el concurso interdisciplinario de endodoncistas, periodoncistas, rehabilitadores orales y laboratoristas dentales. El Síndrome de Colapso de Mordida Posterior (SCMP) bilateral observado se destaca por su gravedad e importancia. El objetivo de este trabajo fue describir de forma integral el diagnóstico y plan de tratamiento en casos complejos realizados con prostodoncia parcial fija en rehabilitación oral y presentar un caso clínico de rehabilitación compleja. [Cabrera JC. Rehabilitación oral con prostodoncia parcial fija: reporte de un caso complejo de arco cruzado. Ustasalud 2012; 11: 45 - 53]
\end{abstract}

Palabras clave: Rehabilitación oral, Prótesis parcial fija, Síndrome de Colapso de Mordida Posterior.

\section{ORAL REHABILITATION WITH FIXED PARTIAL PROSTHODONTICS IN THE COMPLEX CASE OF CROSS ARCH: CASE REPORT}

\begin{abstract}
It is a challenge for rehabilitation restores lost function and aesthetics stomatognathic system. For complex treatments, oral rehabilitation plays a key role in the diagnosis and ensures that objectives are met within academic and practical settings. This report presents a case of complex features restored by fixed partial prosthodontics requiring interdisciplinary participation of endodontists, periodontists, oral rehabilitation and dental laboratory technicians. The Collapse of Posterior Teeth (CPT) reported stands out for its seriousness and importance. The aim of this report was to describe comprehensively the diagnosis and treatment planning in a complex case performed with fixed partial prosthodontics in oral rehabilitation and present a clinical case of complex rehabilitation.
\end{abstract}

Key words: Oral rehabilitation, Fixed partial denture, Collapse of posterior teeth.

Recibido para publicación: 6 de enero de 2012. Aceptado para publicación: julio 2 de 2012.

\section{INTRODUCCIÓN}

La rehabilitación oral como especialidad de la odontología tiene un importante reto al reestablecer de forma integral tanto la función como la estética del sistema estomatognático que puede haberse perdido por patologías pasadas, traumas o accidentes y sus secuelas. ${ }^{1}$ Cuando se intenta rehabilitar un paciente se debe ir más allá del simple pensamiento de colocar prótesis en su boca, se debe pensar en recuperar y recomponer todo el sistema y utilizar para ello, todo el conocimiento y experticia que el profesional pueda poseer y no sólo en lo que se refiere a su especialidad propiamente dicha sino al trabajo en conjunto con otras especialidades. ${ }^{1} \mathrm{El}$ especialista en rehabilitación oral debe conocer tanto de Periodoncia como de Endodoncia, así como de Ortodoncia y de cirugía máxilofacial, esto no quiere decir que realice todos estos procedimientos, sino que debe conocer de forma profunda sus principios, formas de diagnóstico y alternativas de tratamiento ya que en la mayor parte de casos de rehabilitación oral se requiere la interdisciplinariedad. ${ }^{1}$ Todo lo anterior tiene como propósito realizar un tratamiento de rehabilitación integral que cubra todas las expectativas del paciente tanto a nivel funcional como estético. ${ }^{1}$

Por esto, la base de todo tratamiento exitoso debe iniciar con un diagnóstico correcto, es allí donde el especialista debe prestar mayor atención y cuidado, debe juntar toda su experiencia y conocimiento de manera ordenada apoyándose en todas las ayudas diagnósticas para llegar a conclusiones precisas, que lo faculten en el desarrollo de un plan de tratamiento acertado y predecible para sus pacientes. ${ }^{2}$ 
De acuerdo con Giglio y Nicolosi se debe iniciar el diagnóstico con la apertura de la Historia Clínica que no es más que la narración escrita clara, precisa y detallada de todos los datos relativos del paciente y continuar con la inspección, auscultación y percusión del mismo, ${ }^{2}$ de igual forma se debe solicitar siempre una radiografia panorámica, juego periapical, fotos extra e intra-orales y modelos de estudio montados en un articulador semiajustable..$^{3-6}$

Generalmente, los tratamientos de rehabilitación oral complejos se deben protocolizar de acuerdo con las fases de tratamiento propuestas por Kumar y Lum al iniciar con una fase sistémica en donde se prioriza la atención en enfermedades crónicas tales como diabetes, cáncer, enfermedades cardíacas que puedan, por su importancia o gravedad, hacer variar el plan de tratamiento propuesto ya sea en su fase de adecuación como en la de restauración.? Se continúa con la fase de urgencia, de ser requerida, en la que se prioriza la atención en tratamientos por dolor agudo ya sean extracciones o endodoncias para entrar a la fase de adecuación en la que se realizan todas las acciones requeridas para dejar listo al paciente con la finalidad de recibir su tratamiento protésico. $^{7-9}$

Esta etapa finaliza con la confección de las preparaciones dentales realizadas a los dientes pilares seleccionados de acuerdo con el diseño protésico con previa evaluación de los principios biomecánicos clásicos en Prótesis Parcial Fija que son: proporción corono radicular, configuración radicular y la Ley de Ante. ${ }^{3}$ Las preparaciones dentales deben cumplir con los requisitos de retención y resistencia tales como altura gingivoclusal mínima, convergencia oclusal adecuada y tipo de línea terminal; ${ }^{10}$ luego se procede a temporalizar según los requisitos estéticos y funcionales como color, tamaño y forma adecuada, un perfil de emergencia idóneo, superficie lisa y brillante que permita mantener la salud de los tejidos blandos y duros así como la posición de los dientes pilares hasta la cementación de las prótesis definitivas. ${ }^{11}$

La siguiente fase es la restaurativa que se inicia con la confección de un encerado estético para permitir, tanto al paciente, al profesional como al técnico laboratorista, tener una idea precisa de cómo debe quedar la prótesis definitiva; de este encerado se realizará su respectivo índice para que el laboratorio encere la estructura metálica y realice las compensaciones necesarias, donde luego de elaborada la estructura metálica se pueda agregar la porción estética o sea la cerámica. ${ }^{12}$

La impresión se debe tomar con un previo desplazamiento gingival el cual se puede realizar de varias maneras pero la más común es por medios mecánicos como el uso de hilo separador; ${ }^{13}$ al estar listo lo anterior se procede a seleccionar el material para impresión que será un elastómero, generalmente tipo polivinilsiloxano, seleccionado por sus propiedades de copiado, estabilidad dimensional y variedad de consistencias; ${ }^{14}$ cuando se habla de polivinilsiloxano se suele tomar la impresión con dos consistencias, una liviana para que copie el fondo de surco y la línea terminal y la masilla para el resto del arco, así mismo, se debe seleccionar la técnica de impresión de una sola o doble mezcla, la decisión de tomar una u otra radica en la comodidad y experiencia que tenga el operador. ${ }^{14}$

Los demás pasos pertenecen al laboratorio dental, donde se realizará el vaciado de tres modelos de la impresión enviada: uno para confeccionar los troqueles individuales, otro para montar en articulador completo y un tercero de reserva; de los dos primeros se hará el encerado para elaborar la estructura metálica en casos de realizar una prótesis metal cerámica. ${ }^{15}$ Una vez colada la estructura se probará en boca sobre todo en aspectos como estabilidad y adaptación, aprobada la estructura retorna al laboratorio para colocar la cerámica y realizar la prueba final donde se ejecutan las correcciones estéticas necesarias y se procede a cementar, ${ }^{15}$ estos tipos de restauraciones tan complejas donde seguramente se involucran prótesis de arco cruzado y más de diez o doce unidades requerirán al final una placa estabilizadora que el paciente debe usar de forma nocturna para proteger el tratamiento realizado. Este reporte de caso describe de forma integral el diagnóstico y plan de tratamiento de un caso complejo con prostodoncia parcial fija.

\section{REPORTE DE CASO}

Paciente de sexo femenino de 52 años de edad, sin compromiso sistémico que asistió a las Clínicas Odontológicas de la Universidad Santo Tomás en julio de 2008. Su motivo de consulta fue "quiero cambiarme la prótesis de arriba". Se realizó la historia clínica completa que incluyó modelos preliminares en yeso tipo III; además, se procedió a tomar la relación bicondilo-maxilar de la paciente para su respectivo montaje en articulador semiajustable y así realizar el análisis de oclusión. ${ }^{12}$ La formulación del diagnóstico se apoyó en la radiografía panorámica, un juego periapical y fotos intra y extraorales. Los hallazgos fueron:

\section{Exámenes}

En el análisis craneal y facial se encontró que la paciente era de tipo leptoprosópico y dolicocefálico y presentaba un perfil cóncavo; su pómulo izquierdo más alto que el derecho, ala de la nariz izquierda y 
comisura labial izquierda en posición más superior que la derecha, comisura derecha invertida y levognátismo. En el análisis de tercios verticales se observó que el tercio superior estaba disminuido con respecto a los tercios medio e inferior; en el análisis de los quintos faciales no se encontró paralelismo de los mismos, ni paralelismo entre planos bipupilar, nasal y comisural (Figura 1); se le solicitó a la paciente que se colocara su prótesis mucosoportada para evaluar su línea de sonrisa labial y se encontró una línea de sonrisa alta ya que expone el $100 \%$ de sus dientes y una banda de encía (Figura 2); ${ }^{16}$ también, se identificó la presencia de corredor bucal izquierdo mayor que el derecho, el borde superior del labio inferior con respecto a los bordes incisales de dientes anteriores no estaba en concordancia y los caninos contactaban con el labio inferior (vestibularizados).

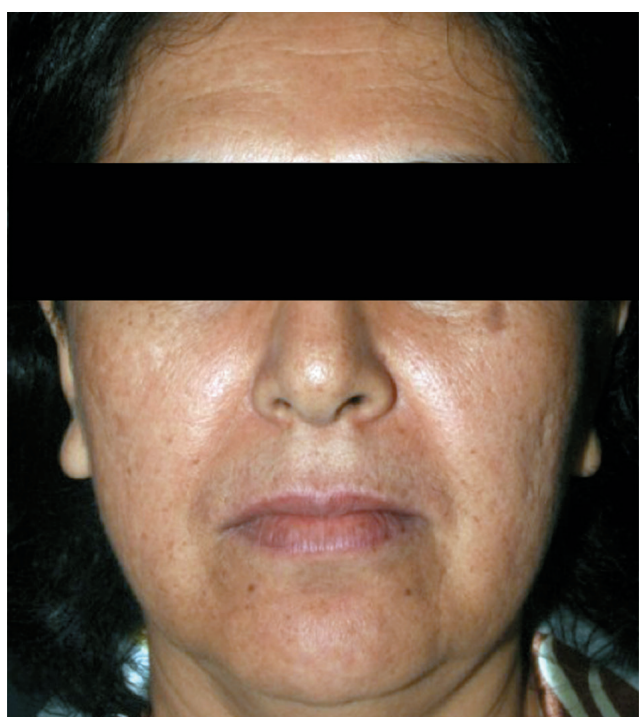

Figura 1. Estado facial de la paciente

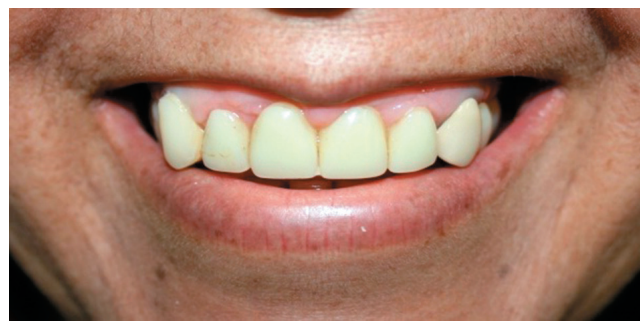

Figura 2. Línea de sonrisa labial

Con respecto a la oclusión de frente se observó ausencia de dientes antero superiores, falta de soporte posteroinferior, facetas de desgaste, malposiciones dentarias (gresiones, rotaciones y versiones); en oclusión derecha se identificó una Clase III canina de Angle, ausencia de primeros molares, curva de Spee aumentada y crecimiento alveolar del segmento de premolares. En oclusión izquierda se identificó una Clase II canina de Angle, ausencia de primeros molares, curva de Spee aumentada y crecimiento alveolar del segmento del segundo premolar. Con relación al estado de la mucosa se encontró inflamación de la mucosa del paladar probablemente a causa de la prótesis mucosoportada respecto al tejido gingival, ${ }^{17}$ así como inflamación gingival asociada a placa bacteriana (Figura 3). ${ }^{18}$
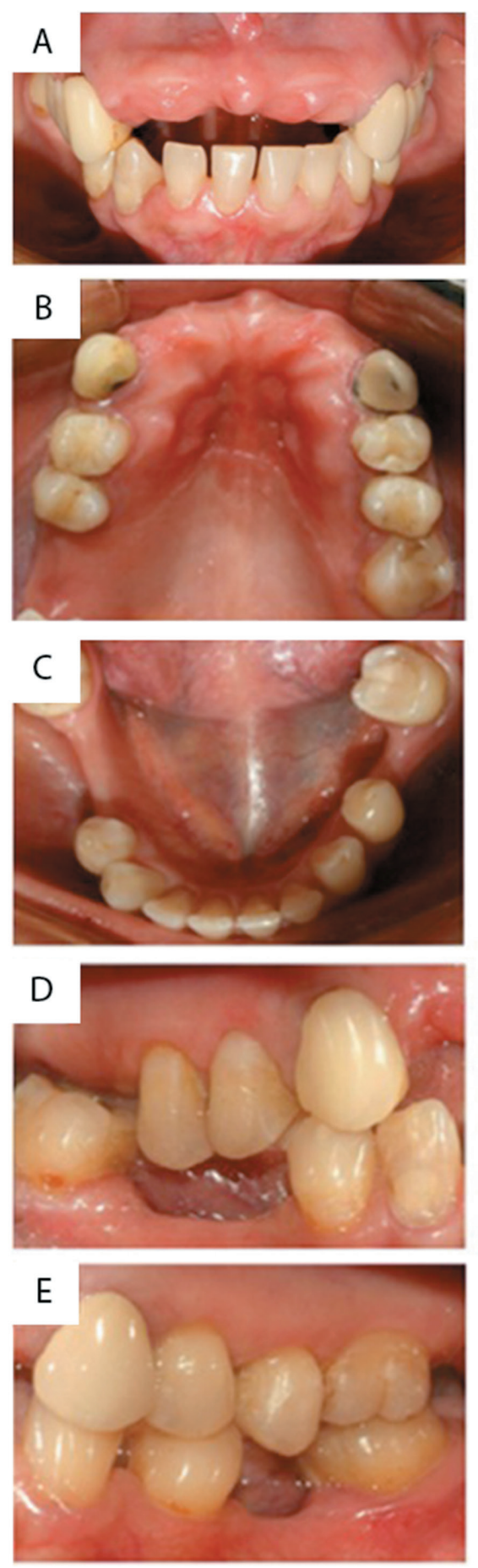

Figura 3. Características clínicas de la cavidad bucal de la paciente. A. Ausencia de dientes anterosuperiores. B. Arco dental del maxilar superior, presencia de mucositis protésica. C. Arco dental del maxilar inferior. D. Oclusión derecha. E. Oclusión izquierda. 
Otros hallazgos fueron la desadaptación de restauraciones en los dientes 13 y 23 donde se identificó la presencia de inflamación gingival y en los dientes 24 y 14. En el análisis de la radiografia panorámica se pudo observar asimetría en formas condilares, neumatización de senos derecho e izquierdo y disminución de la altura de la cresta ósea en ambos maxilares (Figura 4).

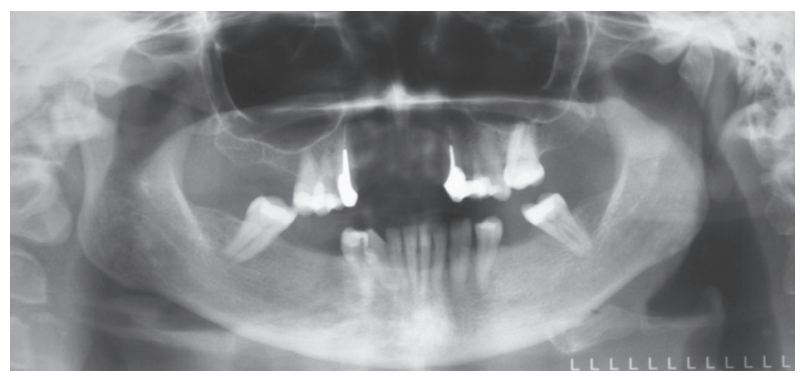

Figura 4. Radiografía panorámica

Los hallazgos de la radiografia periapical del 13 incluyó una zona radiopaca en corona y mitad de la raíz compatible con un retenedor intrarradicular, zona radiopaca en tercio apical compatible con un tratamiento de conducto, presencia de una zona radiolúcida entre retenedor y tratamiento de conducto compatible con una interfase entre los dos elementos, ensanchamiento del ligamento periodontal por mesial y pérdida ósea del mismo lado y una relación corona radicular de 1:2 (Figura 5).

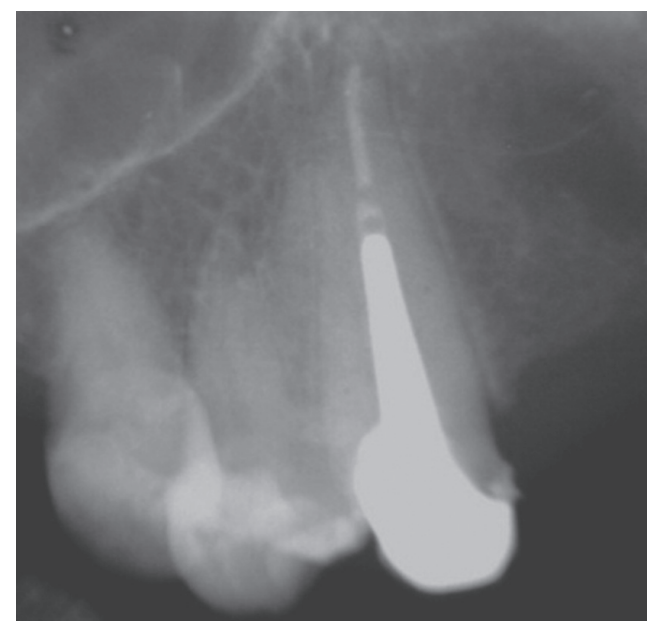

Figura 5. Radiografia periapical de dientes postero-superiores derechos

Con respecto al 23 , se identificó una zona radiopaca en corona y mitad de la raíz compatible con un retenedor intrarradicular, zona radiopaca en tercio apical compatible con un tratamiento de conducto: se observó igualmente una interfase entre los dos materiales y una zona radiolúcida a nivel mesial coronal compatible con una desadaptación del re- tenedor intrarradicular, también se observó ensanchamiento del ligamento periodontal y una relación corono radicular de 1:2. ${ }^{8}$ En el 24 se evidenció una zona radiopaca en la corona compatible con una restauración en resina, zona radiolúcida debajo de la anterior compatible con caries por filtración, en relación con el conducto radicular, presentó una relación corono radicular de 1:1 (Figura 6). Por último, en el 43 se observó la presencia de una zona radiopaca a nivel coronal compatible con una restauración en resina y, a su vez, una zona radiolúcida compatible con caries dental (Figura 7). ${ }^{8}$

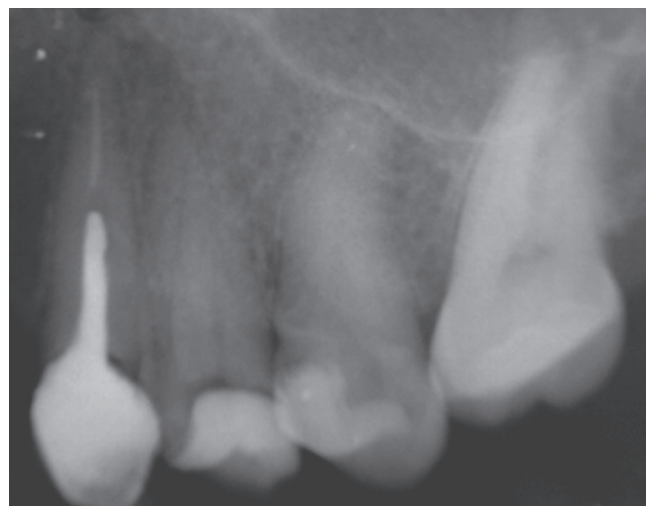

Figura 6. Radiografia periapical de dientes postero - superiores izquierdos

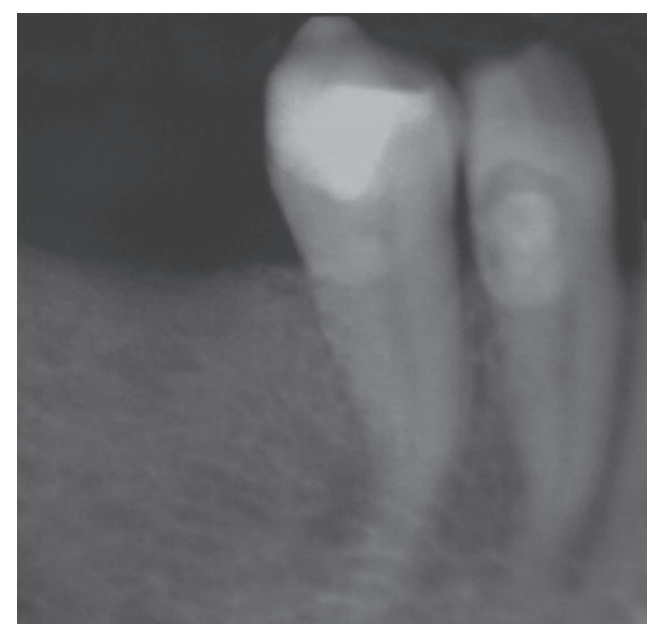

Figura 7. Radiografía periapical de dientes postero - inferiores derechos

\section{Diagnóstico}

En cuanto a la clasificación psicológica del paciente basada en la de M. M. House se encontró que la paciente era un tipo de paciente ideal ya que apreciaba lo que per se hacía por ella, hacía esfuerzos para asegurar el éxito del tratamiento, era una paciente fácil de tratar, confiaba en el tratamiento propuesto y consideraba que lo que el profesional decidía para ella era lo mejor. ${ }^{19}$ 
Presentó caries dental cavitacional activa en la zona mesial del 24 y del 25, oclusión traumática caracterizada por facetas de desgaste en zona anterosuperior e inferior, abfracciones en zona de caninos y premolares inferiores, malposiciones dentales, disminución de la cresta ósea a nivel de los dos maxilares y falta de soporte posterior. ${ }^{12}$ Por otra parte, presentó Síndrome de Colapso de Mordida Posterior (SCMP) bilateral caracterizado por pérdida de la integridad diente-diente, pérdida de soporte posterior, migración dental, cambios en el plano de oclusión y pérdida de la dimensión vertical. ${ }^{20}$

En relación con los espacios edéntulos localizados en el maxilar superior entre los dientes 13 y 23, presentó una clasificación III de Seibertasí como los espacios edéntulos inferiores localizados entre los dientes 37 y 34 y 47 y $44,{ }^{21}$ con respecto al diagnóstico periodontal la paciente presentó una gingivitis generalizada asociada a placa bacteriana; ${ }^{18}$ con relación a la mucosa del paladar se pudo diagnosticar una mucositis a causa del uso de una prótesis mucosoportada donde se presentó una inflación crónica en la zona de contacto con la prótesis, este tipo de lesiones son comunes en más del $60 \%$ de portadores de este tipo de aparatología y su etiología más usual es la falta de higiene oral. De acuerdo con la clasificación de Ceballos, este caso resultó ser una mucositis protésica grado II ya que presentó hiperemia de la mucosa con alisamiento y atrofia. ${ }^{17}$

Con relación al diagnóstico endodóntico este se centró en la situación de los dientes 13 y 23, cada uno con un tratamiento endodóntico previo y un núcleo intrarradicular, la situación ameritaba un análisis profundo ya que éstos serían los pilares de la futura prótesis parcial fija. Dentro de los hallazgos se encontró ambos retenedores cortos, los cuales no cumplían con la longitud de $2 / 3$ radiculares exigida para responder adecuadamente ante las fuerzas y cargas oclusales; así mismo se observó una interfase amplia entre el tratamiento endodóntico y el núcleo intrarradicular.

Además de lo anterior, en el 23 se observó una zona radiolúcida por distal compatible con desadaptación del retenedor intrarradicular y posible caries dental, el tratamiento endodóntico presentaba una menor calidad en el selle y adaptación por lo que se procedió a realizar una anamnesis endodóntica. Según lo expuesto por Chandra se procedió a preguntar a la paciente por el tiempo de realizado de los tratamientos de conducto que resultó ser mayor a los doce años, durante el último año el diente 23 había presentado molestias a nivel periapical con una duración de dos a tres segundos durante cuatro veces en ese último año. ${ }^{22}$

\section{Plan de tratamiento}

Se tuvieron en cuenta las consideraciones de Jacobs que sugieren que para acercarse más al éxito en un plan de tratamiento en casos complejos se debe observar: ${ }^{1}$

1. Expectativas del paciente

2. Tolerancia del paciente

3. Habilidades del clínico

4. Ambiente oral (higiene)

5. Periodontal y oclusión

6. Endodoncia

7. Biomecánica

En cuanto a las dos primeras, la paciente tenía unas expectativas adecuadas y una alta tolerancia a sabiendas de que era un tratamiento largo y dispendioso; en relación con las habilidades del clínico, la paciente se encontraba en un ambiente académico rodeada por docentes expertos y estudiantes profesionales capaces de cumplir con sus expectativas, por otra parte, el ambiente oral de la paciente era bueno debido a que su higiene bucal era aceptable y desde un principio comprendió que debía mejorar para colaborar en su éxito. Con respecto al periodonto, todos los pilares para las prótesis fijas presentaban una proporción corono-radicular adecuada, buen estado periodontal aunque requerían casi en su totalidad un retenedor intrarradicular para ayudar a retener las prótesis requeridas, lo que conlleva al punto siguiente: la endodoncia.

De los diez pilares de la prótesis seleccionados en un principio para el diseño protésico se requirieron ocho para tratamiento endodóntico, todo esto condujo a pensar que el último punto la biomecánica de estos diseños protésicos debía ser óptima para evitar que se fracturaran los pilares y el tratamiento fracasara así como para que un adecuado esquema oclusal ayude a distribuir las fuerzas entre todos los pilares. ${ }^{3}$

En la fase higiénica y periodontal se realizó instrucción en higiene oral, motivación, control de placa bacteriana, alisado y raspaje radicular y retiro de las coronas del 13 y 23. En este caso en particular, la fase de adecuación fue la más importante y la más larga ya que fue necesario muchas citas y diferentes tratamientos interdisciplinarios para que la cavidad bucal estuviera lista para recibir el tratamiento restaurador final. Esta fase se dividió en tres:

A. Fase endodóntica: se decidió realizar tratamientos de conductos en el 24 y como parte de los requerimientos para realizar el tratamiento prostodóntico en los dientes 14, 15, 25,37 y 47 . Se decidió realizar la extracción del retenedor intrarradicular 
del 13 mediante ultrasonido y dejar el retenedor intrarradicular del 23; se procedió a realizar un retratamiento endodóntico del 13. El primer premolar inferior derecho (44) no estuvo desde un principio como diente candidato a recibir tratamiento endodóntico ya que se encontraba sano pero en el trascurso del tratamiento la paciente reportó una odontalgia cada vez más intensa por lo que se decidió realizar tratamiento de conducto para no arriesgar la totalidad del éxito del tratamiento. ${ }^{8}$

B. Fase periodontal: uno de los mayores retos en este tratamiento, fue la nivelación de planos oclusales ya por el SCMP bilateral bastante marcado que presentaba la paciente (Figura 8), ${ }^{20}$ por lo tanto, requería de forma imperiosa nivelar las curvas y planos oclusales para una correcta cinemática mandibular, mejorar la oclusión y la estética. Para iniciar este proceso se decidió realizar alargamientos coronales en $13,14,15,23,24$ y 25 y cuñas distales en 37 y 47 sin sacrificar la relación corono-radicular y se respetó el espacio biológico de cada diente para, posteriormente, realizar unas preparaciones adecuadas (Figura 9). ${ }^{10}$

De igual forma se realizó una cirugía estética mucogingival en el sector anterior con el propósito de nivelar la mucosa y crear nichos gingivales que simularan las papilas interdentales a nivel de los cuatro pónticos anteriores, este procedimiento como su nombre lo indica es puramente estético y tiene como finalidad darle realismo a la prótesis dental definitiva (Figura 10). ${ }^{23}$
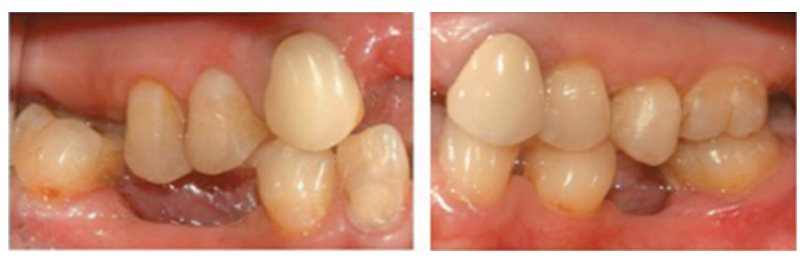

Figura 8. Síndrome de Colapso de Mordida Posterior bilateral

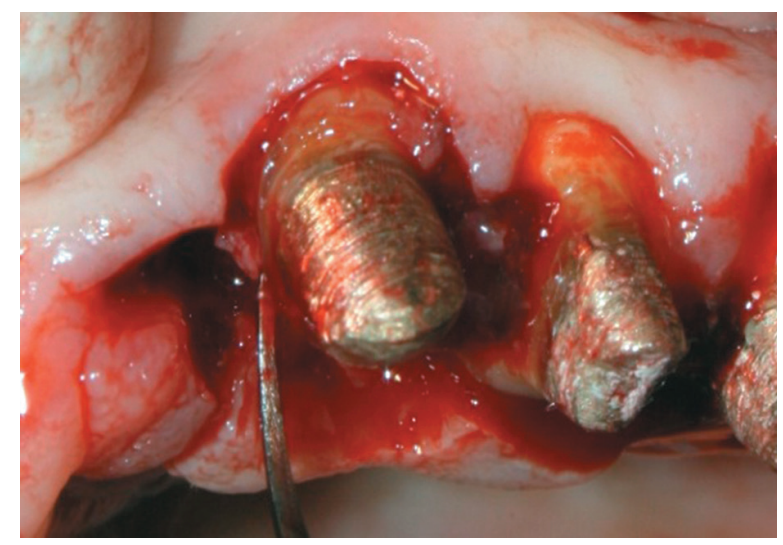

Figura 9. Alargamientocoronal

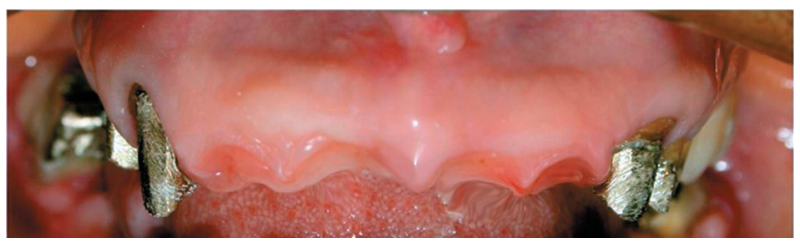

Figura 10. Cirugía estética mucogingival en el sector anterior con el propósito de nivelar la mucosa y crear nichos gingivales

Fase de temporalización: una vez realizados los procedimientos periodontales y endodónticos necesarios, se inició la fase de temporalización definitiva en la que se prepararon todos los dientes pilares (13, $14,15,23,24,25,34,37,44$ y 47$)^{11}$ y se usó como línea terminal un chamfer pesado. El proceso de temporalización se realizó con el encerado elaborado previamente, la temporalización se hizo mediante la técnica de la matriz de silicona en la que se toma una matriz en silicona al encerado y ya en boca con los dientes preparados, se procede a rellenar la matriz con resina acrílica del color seleccionado, se retiran excesos, se le da el perfil de emergencia adecuado con discos y fresas de laboratorio, se pulen y brillan. Se tiene el cuidado de fabricar pónticos ovales que presionen el tejido blando sobre los nichos construidos quirúrgicamente para conservar y mejorar las papilas interdentales se ajustan y se chequea oclusión (Figuras 11 y 12). ${ }^{11}$

Fase correctiva: en este caso se realizaron tres distintas prótesis parciales fijas, una en el maxilar superior de arco cruzado de diez unidades con seis pilares y cuatro pónticos y dos inferiores una de tres unidades que abarcaba del 37 al 35 con dos pilares y un póntico; la última de cuatro unidades del $47 \mathrm{al}$ 44 con dos pilares y dos pónticos. Para el caso en particular se seleccionó una prótesis metal cerámica, el metal escogido fue una aleación de metal base níquel cromo. ${ }^{15}$

Una vez definido y confirmado el diseño protésico, se procedió a tomar los registros de oclusión, la llave de oclusión para mantener la dimensión vertical adecuada y alcanzada en la fase de provisionalización y la toma de la impresión. Para la toma de los registros interoclusales se procedió a realizar un juego de plato bases con rodetes en resina acrílica rosada y se realizó una llave de oclusión con patter resin para mantener la dimensión vertical ajustada debido a que no había una estabilidad adecuada por las preparaciones realizadas y las numerosas ausencias dentales. ${ }^{24}$

Para la toma de impresión definitiva se seleccionaron los siguientes materiales: ${ }^{14}$ polivinilsiloxano de consistencia liviana y de masilla, hilo separador triple cero y doble cero, hemostático, pistola para aplicar la silicona liviana y cubetas stock superior e inferior talla $\mathrm{M}$. 
En primer lugar se anestesiaron los dientes pilares para iniciar la colocación del hilo separador más delgado, el triple cero, el cual se introdujo dentro del surco gingival con ayuda de un instrumento específico para esta actividad; una vez llevado el hilo separador a todos los dientes pilares se procedió a re-preparar los mismos y dejar la línea terminal subgingival a $0,5 \mathrm{~mm},{ }^{13}$ por último con una fresa especial se pulieron las preparaciones, se eliminó así cualquier estría o marca. Una vez conseguido lo anterior se colocó un hilo separador más grueso, el doble cero, en este caso se esperaron ocho minutos para iniciar la toma de impresión; ${ }^{13}$ la técnica utilizada para la toma de impresión fue de doble mezcla $\mathrm{y}$ un solo paso (Figura 13). ${ }^{14}$

De estas impresiones se obtuvieron tres modelos en yeso tipo V: el primero para realizar troqueles individuales mediante la técnica del dowell pin, el segundo para ser montado en articulador y el tercero como reserva. Se realizó un encerado estético para probar en boca y tener un acercamiento definitivo a las prótesis fijas, este se probó en boca y se le hicieron los cambios correspondientes, con ese resultado final se tomaron dos índices o matrices (superior e inferior) en silicona de condensación. En el laboratorio se enceró la estructura de las prótesis fijas colocando los índices periódicamente para hacer las compensaciones necesarias en las zonas que lo requerían, una vez terminado este proceso se procedió a revestir el encerado y posteriormente a colarlo.

Una vez terminado este proceso se recortó, pulió y brilló y se probó en boca para analizar la estabilidad y adaptación marginal. Después de una cuidadosa observación se identificó el cumplimiento de los requerimientos y se envió para realizar la soldadura entre incisivos centrales. ${ }^{15}$ Se probó nuevamente, se encontró bien y se envió al laboratorio para la colocación de la cerámica; el laboratorio colocó la cerámica color $\mathrm{A} 3$ en tercio incisal y medio y un A3,5 en cervical, se entregó a prueba, se realizaron algunos cambios estéticos menores y se dio la orden de glacear y cementar en forma definitiva con cemento de resina autoadhesivo autograbador (Figura 14). ${ }^{15}$

Por último se realizaron dos controles para observar alguna anomalía, problema o dolor y se realizó una placa estabilizadora para proteger la aparatología colocada en boca. ${ }^{7}$

\section{DISCUSIÓN}

Está claro que la rehabilitación oral es más que simplemente colocar prótesis en un paciente determinado para reemplazar algunos dientes perdidos, implica un esfuerzo mayor y de forma integral para comprender las consecuencias de las patolo- gías orales y traumas que han llevado al sistema estomatognático a requerir de un tratamiento altamente especializado e integral para retornar sus antiguas funciones y estética; ${ }^{1}$ este proceso se inicia desde el diagnóstico que debe realizarse con sumo cuidado y dedicación dándole la importancia que se merece y otorgándole el tiempo suficiente para su interiorización mediante la solicitud de los exámenes necesarios para complementar y profundizar los mismos ${ }^{2}$.

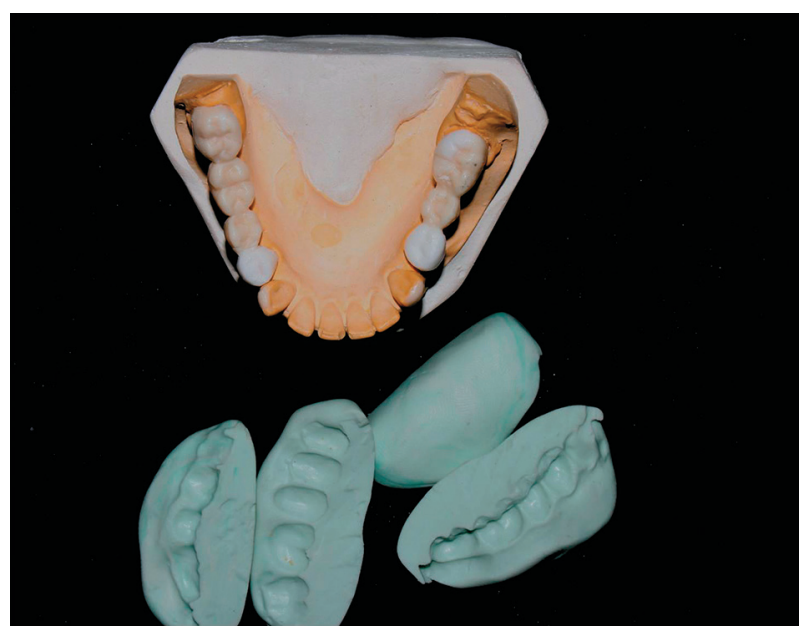

Figura 11. Matriz de silicona para obtener las provisionales

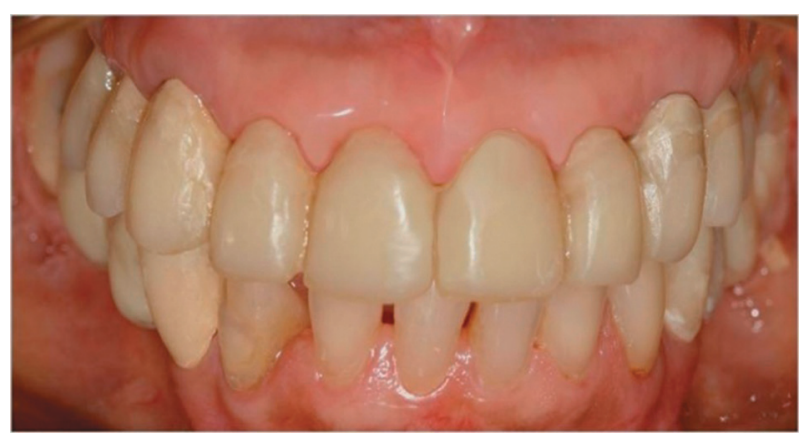

Figura 12. Temporalización

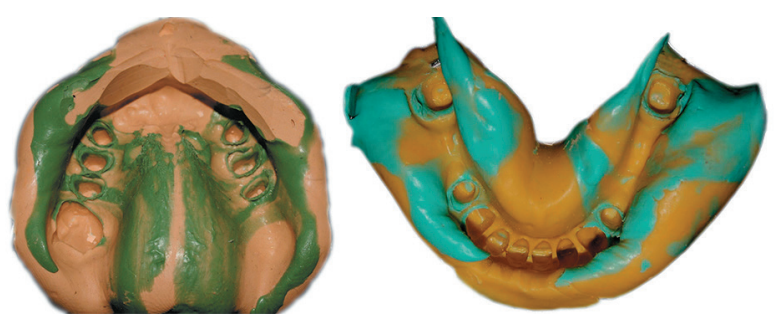

Figura 13. Impresiones definitivas superior e inferior

Posiblemente uno de los problemas más complejos en rehabilitación oral es la restauración de los casos en los que existe una falta de soporte posterior que conlleva a un SCMP. Éste se caracteriza por: ${ }^{20}$ 

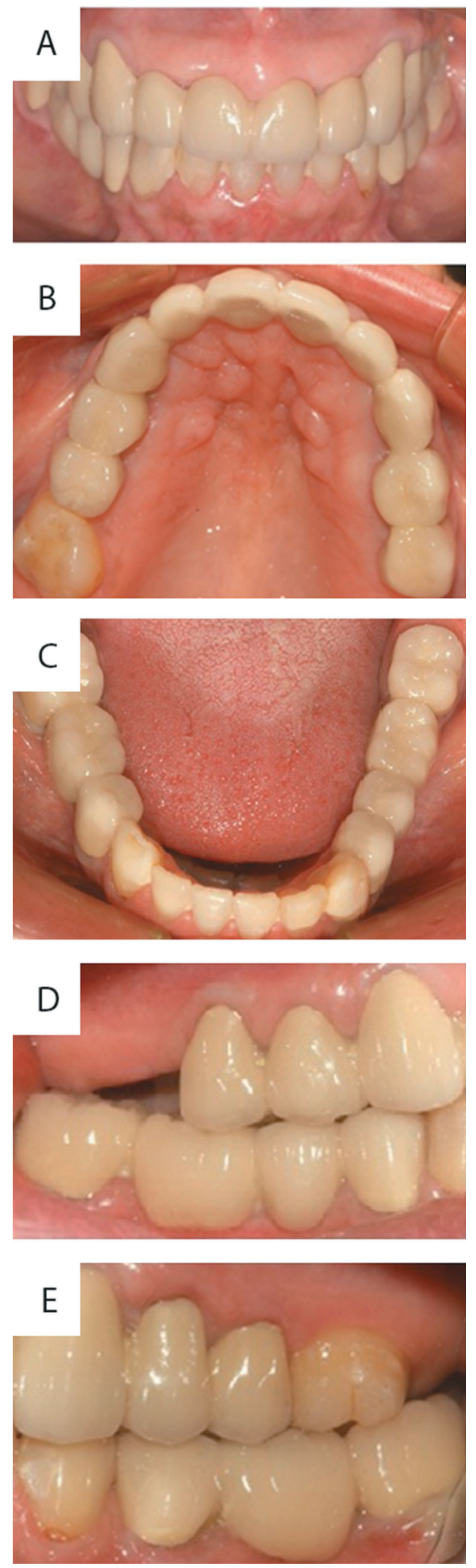

Figura 14. Las prótesis parciales fijas fueron cementadas en forma definitiva con cemento de resina autoadhesivo autograbador. A. Oclusión de frente. B. Arco superior. C. Arco inferior. D. Oclusión derecha. E. Oclusión izquierda.
- Pérdida de la integridad arco

- Pérdida de la integridad diente-diente

- Pérdida del soporte posterior

- Migración dental

- Interferencia en balanza, trabajo y protrusiva

- Cambio de los planos de oclusión

- Pérdida de la orientación funcional raíz eje

- Pérdida de la dimensión vertical

- Desequilibrio muscular en labio, carrillo, lengua y aparato masticatorio

La pérdida de integridad arco-arco se caracteriza por cambios dentales y reposición anterior de la mandíbula, ${ }^{20} \mathrm{y}$ la pérdida de integridad dientediente se destaca por la pérdida de la armonía meso distal y vestíbulo lingual en los arcos opuestos. La paciente del presente caso mostró una pérdida de la integridad diente-diente así como pérdida de soporte posterior con migración dental acompañada de ausencia de la guía anterior y dientes anteroinferiores en abanico. Adicionalmente, presentó alteración de contactos en el lado de balanza tanto en izquierda como en derecha; una de las características más sobresalientes del SCMP fueron los cambios en los planos de oclusión, los cuales en un principio se observaron dramáticos al formar unas curvas de Spee bastante agudas que no hacían predecir un buen pronóstico, esto, tal vez, fue uno de los mayores retos en su rehabilitación puesto que se pensó en realizarla con prostodoncia fija y lo primero que se debía hacer era pensar en la nivelación de los planos oclusales a través de las preparaciones dentales pero el encerado diagnóstico mostró muy rápidamente que esto no sería suficiente. Por eso fue tan importante la fase de adecuación que permitió nivelar las curvas y mejorar el plano oclusal para eliminar las interferencias en el lado de balanza derecho e izquierdo. ${ }^{5,8,9}$

Otro signo importante en el SCMP es la pérdida de la orientación funcional raíz-eje. Los dientes posteriores a los espacios edéntulos tienden a migrar hacia mesial y a sufrir una inclinación hacia ese mismo lado observándose dientes inclinados con pérdida de su orientación corono-radicular, ${ }^{3,20}$ su solución depende del grado de inclinación que posea la pieza dental. Cuando es mayor a $30^{\circ}$ se considera la ortodoncia para paralelizar las raíces, si es menos de tal angulación se puede optar por hacer una corona telescópica como lo sugiere Shillimburg o simplemente hacer una preparación extra-coronal acompañada de una cuña periodontal por distal para alargar la preparación. En este caso, la inclinación no superaba los $20^{\circ}$ por lo que se realizó el último tratamiento mencionado. ${ }^{3}$ 
Además el SCMP se caracteriza por la disminución de la dimensión vertical del paciente por falta de soporte posterior, exactamente la ausencia de cúspides estampadoras de molares superiores e inferiores; en este caso se restauró la dimensión vertical en la zona posterior con $0,5 \mathrm{~mm}$ con el propósito de ayudar a nivelar planos y dar una solidez estructural a las preparaciones dentales realizadas y a los materiales utilizados para la confección de las prótesis fijas. ${ }^{23}$

Para finalizar es necesario mencionar que la función precede a la estética, todo aquello que se realice para devolver la función será garantía de que la estética podrá tener predecibilidad, de lo contrario, pensar primero en los requerimientos estéticos traerá problemas funcionales que terminarán por afectar también la estética misma. ${ }^{16}$ De acuerdo con este caso, la fase de adecuación cobró una gran importancia para el paciente y es, tal vez, una parte interesante de entender ya que muchas veces se espera que sea la fase restaurativa la más relevante; en estos tipos de tratamientos complejos se requiere de una gran habilidad diagnóstica sin dejar nada a la deriva. Siempre es vital integrar todos los aspectos dentales para beneficio del paciente. ${ }^{2,7}$

\section{Conclusiones}

Un diagnóstico preciso es vital en el buen desarrollo de un tratamiento ya que su integralidad y profundidad pueden hacer la diferencia entre un tratamiento adecuado y uno eximio.

Un plan de tratamiento siempre se debe enfocar mediante un protocolo estándar donde que incluya la fase sistémica, de urgencia, higiénica, de adecuación, restauradora y la fase de mantenimiento.

Un caso exitoso en rehabilitación oral implica muchos aspectos que siempre se deben tener en cuenta: la experticia del operador, su buena comunicación con el laboratorista dental, un buen equipo de trabajo interdisciplinario, el uso de materiales de alta calidad, herramientas de diagnóstico actuales, buen desempeño diagnóstico y conocimiento profundo de la biología del sistema estomatognático.

\section{BIBLIOGRAFÍA}

1. Jacobs D, Steele J, Wassell R. Crowns and extra-coronal restorations: considerations when planning treatment. Br Dent J 2002; 192: 257 - 267.

2. Giglio M, Nicolosi L. Semiologia en la Práctica de la Odontología. Mc Graw Hill-Interamericana. 2000; pp. 3 - 6.

3. Shillingburg HT, Hobo S. Fundamentos de Prostodoncia Parcial Fija. Quintessence Publishing; 2008. Pp. 34 - 39

4. Peck H, Peck S. A concept of facial esthetics. Angle Orthod 1979; 40: $284-371$.

5. Davies S, Gray R. What is occlusion? Br Dent J 2001; 191 : $235-245$.
6. Ngom P, Diagne F, Benoist $\mathrm{H}$, Thiam F. Intraarch and interarch relationships of the anterior teeth and periodontal conditions. Angle Orthod 2006; 76: $236-241$.

7. Kumar C, Lum M. Restoration - General considerations and the pretreatment evaluation. J Calif Dent Assoc 2008; 36: $827-834$.

8. Whitworth J, Walls A, Wassell R. Crowns and extra-coronal restorations: Endodontic considerations: the pulp, the root-treated tooth and the crown. Br Dent J 2002; 192: 315 - 327.

9. Escudero N, García V, Bascones J, Bascones A. Alargamiento coronario, una necesidad de retención protésica, estética y anchura biológica. Revisión bibliográfica. Av Odontoestomatol 2007; 23: 171 -180.

10. Goodacre C. Designing tooth preparations for optimal success. Dent Clin North Am 2004; 48: 359 - 385.

11. Gratton D, Aquilino S. Interim restorations. Dent Clin North Am 2004; 48: 487 - 497.

12. Steele J, Nohl F, Wassell R. Crowns and extra-coronal restorations: occlusal considerations and articulator selection. Br Dent J 2002; 192: 277 - 286.

13. Terry E, Donovan D, Winston W, Chee B. Current concepts in gingival displacement. Dent Clin North Am 2004; 48: $433-444$.

14. Wassell R, Barker D, Walls A. Crowns and extra-coronal restorations: impression materials and technique. $\mathrm{Br}$ Dent J 2002; 192: 679 - 689.

15. Wassell R, Walls A, Steele J. Crowns and extra-coronal restorations: materials selection. Br Dent J 2002; 192: 199 $-211$.

16. Fradeani M. Rehabilitación Estética en Prostodoncia Fija - Análisis Estético. Un Acercamiento Sistemático al Tratamiento Protésico. Quintessence Publishing; 2006.

17. Ayuso R, Torrent J, López J. Estomatitis protésica: puesta al día. RCOE 2004; 9: 657 - 662.

18. Dimitris N. Tatakis, D, Purnima S. Kumar S. Etiology and pathogenesis of periodontal diseases. Dent Clin North Am 2005; 49: 491 - 516.

19. Gamer S, Tuch R, Garcia LT. M. M. House mental classification revisited: intersection of particular patient types and particular dentist's needs. J Prosthet Dent 2003; 89: $297-302$.

20. Bardález R, Armero C, Lira S. Colapso posterior de mordida. La Carta Odontológica 2002; 7: 10 - 14.

21. Escudero N, Lorenzo R, Perea M, Bascones A. Autoinjerto de tejido conectivo para aumento del volumen de tejidos blandos. Indicaciones y aplicación clínica. Av Periodon Implantol 2008; 20: 113 -120.

22. Chandra A, Discuss the factors that affect the outcome of endodontic treatment. Aust Endod J 2009; 35: 98 - 107.

23. Clemens W, Buttel L, Weiger R. Localized alveolar ridge augmentation using a two-step approach with different soft tissue grafts: a clinical report. J Contemp Dent Pract 2008; 9: $99-106$.

24. Turrell A. Clinical assessment of vertical dimension. J Prosthet Dent 2006; 96: 79 - 83. 